\title{
Improvement of procedures for high accuracy micromilling of flat surfaces
}

\author{
A. Banfi, L. Colombo, F. Cacciatore, L. Rebaioli, M. Annoni
}

Dipartimento di Meccanica, Politecnico di Milano, Italy

\begin{abstract}
All the resources involved in micromilling operations (machine tool, tool, fixture, workpiece) have to be accurately managed to improve the final workpiece quality. Special attention has to be paid also to system thermal stability, micromilling process parameters and machining strategies, workpiece and tool measurement accuracy. The present paper proposes an easy and industry-oriented procedure to maximize the achievable workpiece accuracy working in absolute coordinates. Accurate plane surfaces with a strict flatness deviation are machined on a CuNi12Zn30Pb1 benchmark workpiece to demonstrate the procedure effectiveness.
\end{abstract}

Keywords: micromilling, accuracy, touch probe, ANOVA

\section{Introduction}

Micromilling operations involve four fundamental resources (Fig. 1), which need specific features:

- machine tool (A in Fig. 1): thermal and dynamic stability to ensure high accuracy, low CNC sampling times to guarantee chord error reduction [1-5];

- tool (B in Fig. 1): tool geometry (edge radius plays a fundamental role on the minimum uncut chip thickness definition), tool material (ultrafine hard metal grains should be preferred), coating (a large amount of ploughing occurs in micromachining and a suitable coating is needed to reduce friction and built-up-edge) [1-3];

- fixture ( $C$ in Fig. 1): ad hoc fixtures, which let five workpiece faces free, to allow performing the whole working and measuring cycle with only one fixturing operation, thus reducing workpiece errors;

- workpiece: homogeneous materials, at least at the mesoscale, to simplify the process parameters selection [1-2].

A previous research carried out by the authors of the present paper [6-7] highlights that in micromachining operations specific attention has to be paid also to machining strategies (D in Fig. 1) and tool and workpiece measuring strategies ( $E$ in Fig. 1). In particular, the authors developed a warm-up and tool length compensation strategy to manage factors affecting tool length, as machine spindle thermal transients and tool wear (Fig. 2). The results demonstrated how such an approach is able to face tool length variations both in case they are real (depending on tool wear) and fictitious (depending on spindle thermal deformations). A $5 \mu \mathrm{m}$ height variation on the workpiece flat surface was achieved.

The present paper will investigate other factors that affect the workpiece accuracy. For instance, while the design characteristics of machines (e.g. structure dimensions) cannot be changed, calibration and maintenance have to be accurately performed since they play a fundamental role in cutting operations (Section 3). Moreover, theoretical and empirical approaches suggest to reduce cutting forces in order to avoid chatter and tool deflection, which affect the workpiece accuracy. For this reason, the micromilling process parameters should be specifically designed (Section 3).

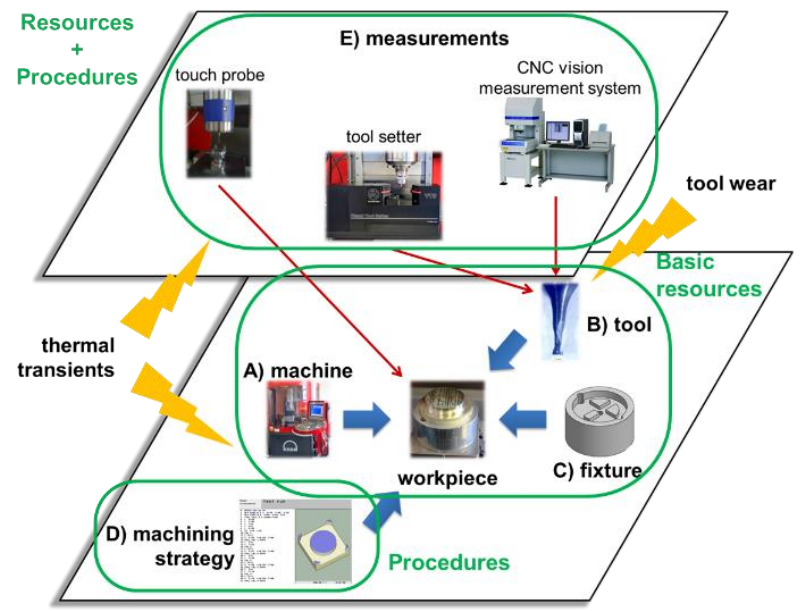

Fig. 1. Resources and procedures involved in a micromilling operation.

Eventually, the complete characterization of measurement sensors is necessary to minimize the workpiece errors. Investigating the factors that affect the measurement scattering allows to design a measurement strategy to increase the accuracy of origin setting and, hence, to reduce workpiece errors (Section 4.2 - 4.3).

In conclusion, the present paper aims at designing and testing an easy and industry-oriented procedure to maximize the achievable workpiece accuracy working in absolute coordinates (i.e. the machine has to reach the same $Z$ position in different time instants and with different tools). This objective is achieved by an accurate management of system thermal behaviour, micromilling process parameters, machining strategies, workpiece and tool measurement accuracy.

The selected case study is simple but industrially significant. Accurate plane surfaces with a strict flatness deviation have been machined on a benchmark workpiece. 


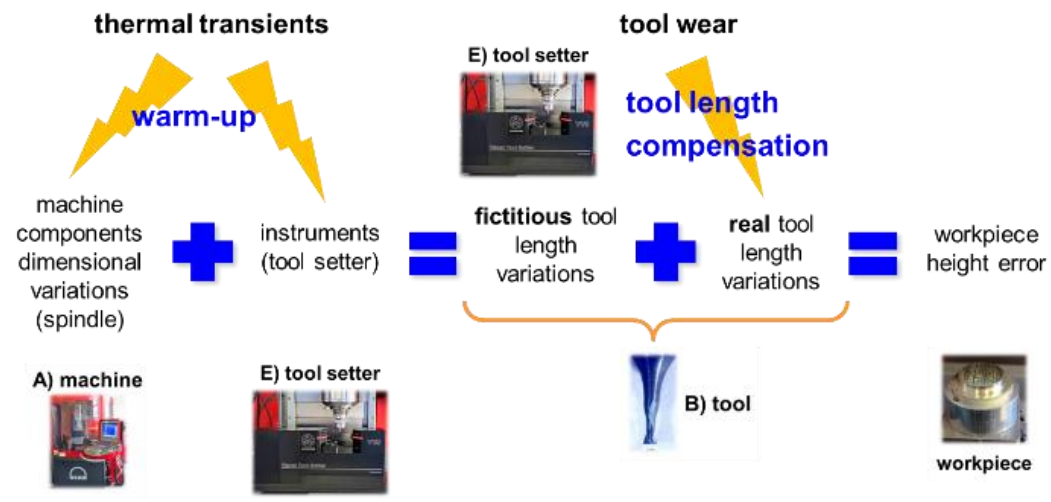

Fig. 2. Factors affecting the workpiece height error.

\section{Objectives}

The case study of this paper is obtaining the maximum accuracy in $Z$ direction on a Cu-Ni12Zn30Pb1 copper-nickel zinc alloy specimen (Fig. 3) working in absolute coordinates.

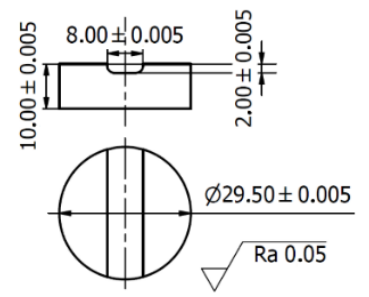

Fig. 3. Benchmark workpiece (CuNi12Zn30Pb1).

The selected case study is significant for industry, in particular for mold micromachining. In fact, even if the workpiece of this study is very simple, accurate machining of flat surfaces in absolute coordinates is very useful when manufacturing molds for process as micromolding, microforming and microextrusion.

\section{Experimental setup}

This Section describes the resources that play a role in the selected micromilling operation.

The whole procedure was designed and tested on the 5-axis Kern EVO ultra precision machining centre (A in Fig. 1) available at "Ml_crolab", the mechanical micromachining laboratory of Department of Mechanical Engineering of Politecnico di Milano. In the present study, the machine was used in 3-axis configuration (nominal positioning tolerance (according to VDI/DGQ 3441) $= \pm 1 \mu \mathrm{m}$ ).

To obtain reliable results, the machine was completely checked and calibrated prior to performing the machining operation described in this paper. Collision effects, axis guide preload losses, axis straightness and squareness errors have been measured by a laser interferometer system.

Regarding the tools ( $B$ in Fig. 1), Table 1 presents the characteristics of the used finishing flat-end mill and the selected cutting parameters.

In the previous paper by the same authors of this study authors [6-7], tool diameter and process parameters were specifically selected to require high machining time, thus focusing on tool wear assessment. In the present research, tool diameter and cutting parameters were chosen to minimize the working time, since working loads introduce a large thermal power with negative effects on the process accuracy. However, looking for optimal machining parameters is not the purpose of this paper.

Table 1. Mill characteristics and constant cutting parameters in finishing.

\begin{tabular}{|c|c|c|}
\hline \multicolumn{3}{|c|}{ Seco 553L030Z30-SIRON-A } \\
\hline Material & $D_{\mathrm{c}}$ & solid carbide \\
\hline Diameter $(\mathrm{mm})$ & $z$ & 3 \\
\hline Teeth number & $v_{\mathrm{c}}$ & 200 \\
\hline Cutting speed $(\mathrm{m} / \mathrm{min})$ & $n$ & 21220 \\
\hline Spindle speed $(\mathrm{rpm})$ & $a_{\mathrm{p}}$ & 0.05 \\
\hline Axial depth of cut $(\mathrm{mm})$ & $a_{\mathrm{e}}$ & 1 \\
\hline Radial depth of cut $(\mathrm{mm})$ &
\end{tabular}

A proper fixture (C in Fig. 1) was designed to hold the workpiece (Fig. 3) during milling operations. The fixture was machined on the Kern EVO machining center from an aluminum block. In this fixture, the workpiece was positioned on three small flat surfaces (pointed by yellow arrows in Fig. 4) and was held in position by the lateral cylindrical surface with the addition of cyanoacrylate adhesive.

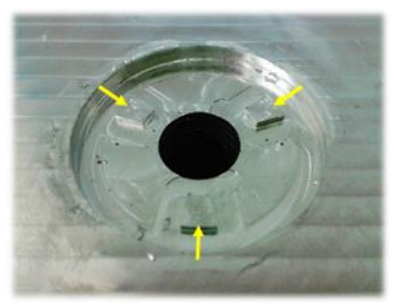

Fig. 4. Fixture detail.

Machining strategies and process parameters (D in Fig. 1) have to be carefully designed to ensure high workpiece accuracy. In order to minimize static and dynamic effects, cutting forces should be kept low. For this reason, smaller chip thickness and depth of cut lead to higher accuracy and surface quality. Fig. 5 shows the surface quality achieved on the present target material with different feeds per tooth and constant $a_{\mathrm{p}}=0.05 \mathrm{~mm}$. A roughness $R_{\mathrm{a}}=0.03 \mu \mathrm{m}$ (stylus profilometer, cut-off length $\lambda_{\mathrm{c}}=0.8 \mathrm{~mm}$ ) has been achieved when machining with a feed per tooth $f_{\mathrm{z}}=0.0025 \mathrm{~mm}$ (Fig. 5b).

The overlap between consecutive passes also affects the final surface quality. Past experience points 
out how, in the selected case study, an overlap equal to $0.3 \cdot D_{\mathrm{c}}$ is the best trade-off between machining time and final quality. Past experience shows also how that, to achieve the best surface quality, compressed air is required to remove chips and to reduce thermal expansion of materials during cutting operations.
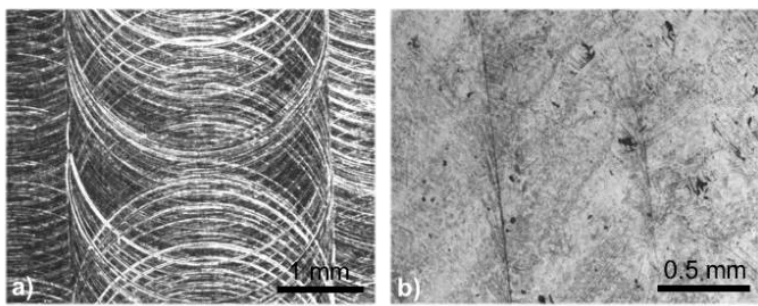

Fig. 5. Surface quality with a) $f_{\mathrm{z}}=0.02 \mathrm{~mm}$, b) $f_{\mathrm{z}}=0.0025 \mathrm{~mm}$ (images acquired by Mitutoyo Quick Vision QVP202).

Regarding the machining strategy, surfaces should be finished in a unique step, with the same origin and tool, to avoid any effects of wear and calibration errors. Sometimes, an additional pass with null depth of cut is useful to reach the target accuracy. Adopting a constant $Z$ axis milling strategy, even during rapid movements, does not improve the workpiece accuracy since it has been verified how the machine control always acts on $Z$ axis keeping its position within $\pm 1 \mu \mathrm{m}$.

The measurement equipment ( $E$ in Fig. 1) includes the Marposs VTS ${ }^{\odot}$ (Visual Tool Setter) that has been used for onboard tool measurements (namely, length, diameter, radial run out). This device has a resolution of $0.1 \mu \mathrm{m}$ and a repeatability of $0.2 \mu \mathrm{m}$ $(2 \sigma)$. Moreover, onboard workpiece measurements have been carried out by the m\&h 32.00-MINI infrared touch probe, which is installed on the Kern EVO machining centre. This device has a repeatability for single-point surface measurement (ISO 230-10:2011) $R_{\text {spt,Z }}=1.2 \mu \mathrm{m}(2 \sigma)$. Eventually, a CNC vision measurement system, Mitutoyo Quick Vision QVP202, has been used for the offboard tool and workpiece surface characterization.

\section{Experiments}

\subsection{System thermal stability}

The machine spindle thermal transient has already been studied [6-7]. A suitable warm-up strategy has been proposed to minimize the thermal transient effects on tool length variations (Fig. 2).

Further experiments demonstrated how the $Z$ axis thermal transient can be split into two components: a short-term component, mainly related to the spindle ( $\approx 2 \mu \mathrm{m}$ in $10 \mathrm{~min}$ ), and a long-term component (up to $20 \mu \mathrm{m}$ in $12 \mathrm{~h}$ ). According to these results, the system thermal equilibrium is ensured by warming up the spindle for 12 hours at $75 \%$ of the working rotational speed. After that, warming up the spindle for 30 minutes at $100 \%$ of the working rotational speed is required to eliminate any transients.

\subsection{Tool measurement accuracy}

Marposs VTS ${ }^{\odot}$ origin has to be set using a calibrated pin prior to each milling operation, otherwise tool measurements can be affected by errors up to $0.02 \mathrm{~mm}$.

\subsection{Touch probe accuracy}

The touch probe accuracy is critical, in particular when finishing cannot be performed in a unique step or a new origin is necessary.

Preliminary tests suggested that the touch probe feed rate and stand-off distance (i.e. the distance between the surface defined by a first rough measurement and the touch probe tip) could affect the final measurement in $Z$ direction. A proper experimental design (Tab. 2) has been selected in order to point out the effects of the aforementioned parameters on measurements in $Z$ direction.

Table 2. Experimental design summary

\begin{tabular}{cccc} 
& \multicolumn{3}{c}{ Levels } \\
Factor & - & $\mathbf{0}$ & $\boldsymbol{+}$ \\
\hline Feed rate $(\mathrm{mm} / \mathrm{min})$ & 50 & 100 & 150 \\
\hline Stand-off distance $(\mathrm{mm})$ & 1 & 10 & 20 \\
\hline
\end{tabular}

Three levels have been considered for the feed rate: $50 \%, 100 \%$ and $150 \%$ of the value suggested by the m\&h 32.00-MINI touch probe manufacturer (100 $\mathrm{mm} / \mathrm{min}$ ). The stand-off distance has been varied between $1 \mathrm{~mm}$, which is a reasonable lower limit, and $20 \mathrm{~mm}$, which is the upper limit for this touch probe.

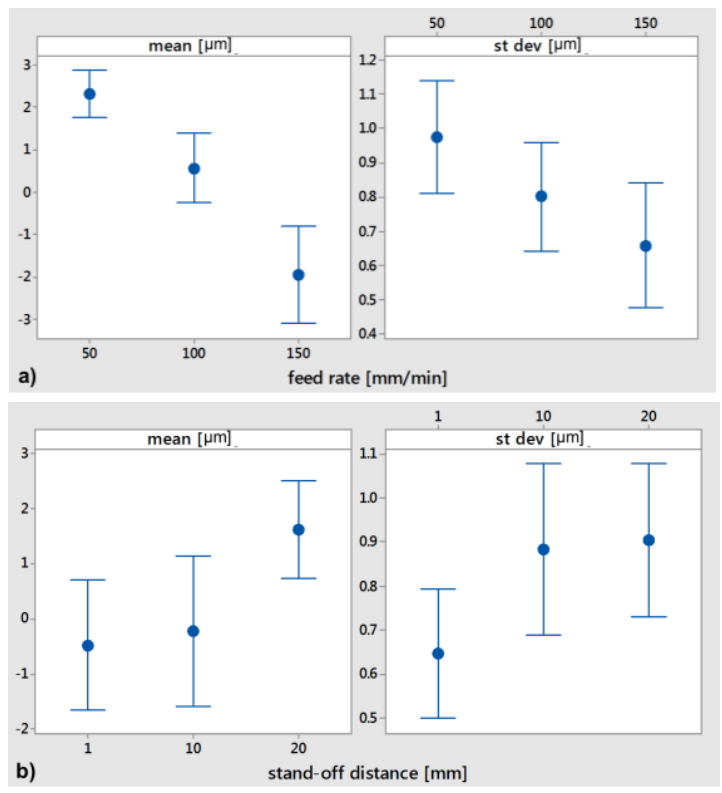

Fig. 6. Interval plots (95\% confidence interval for the mean) of measurement mean and standard deviation against a) feed rate and b) stand-off distance factors.

5 repetitions have been performed for each factor combination (i.e. the measurement in $Z$ direction has been repeated 5 times consecutively) and the 9 experimental conditions have been replicated 6 times. A block factor has been put on replicates to remove any possible nuisance effect of drift over time. The runs have been randomized inside each block. After each block the touch probe has been dismounted from the spindle, mounted again and calibrated on a reference surface, whose $Z$ position is known.

Mean and standard deviation of the 5 measurements in $Z$ direction in each replicate are the 
responses of experimental design (Fig. 6). A two factor (feed rate and stand-off distance) complete model with blocks has been analysed for both responses. The ANOVA results are summarized in Tab. 3 .

The ANOVA p-values (Tab. 3) show how the block factor does not affect any responses. Moreover, the measurement mean value is influenced by both factors and their interaction while only feed rate affects the measurement standard deviation. In conclusion, the experiment suggests that the touch probe must be used through the whole milling operation with the same feed rate and stand-off distance since both the acquired $Z$ value mean and standard deviation are affected by the measurement parameters.

Table 3. ANOVA p-values for mean and standard deviation responses (confidence level $\alpha=5 \%$ ).

\begin{tabular}{|c|c|c|c|}
\hline & \multicolumn{2}{|c|}{ Responses } \\
\hline & & Mean & Std. dev. \\
\hline \multirow{4}{*}{ } & Block & 0.186 & 0.988 \\
\hline & Feed rate $(F)$ & 0.000 & 0.031 \\
\hline & Stand-off distance (D) & 0.000 & 0.058 \\
\hline & $D^{*} F$ & 0.003 & 0.640 \\
\hline & & $\sigma=1.28$ & $\sigma=0.346$ \\
\hline
\end{tabular}

A pairwise comparison among the three level mean values of standard deviation data has been performed by a Tukey's test $(\alpha=5 \%)$. The results point out how there is no significant difference between level mean values for feed rate equal to $100 \mathrm{~mm} / \mathrm{min}$ and $150 \mathrm{~mm} / \mathrm{min}$. Therefore, the higher feed rate of $150 \mathrm{~mm} / \mathrm{min}$ has to be preferred in order to reduce the measurement scattering (Fig. 6a) and also the measuring time. Since stand-off distance does not affect the measurement scattering, the lowest value $(1 \mathrm{~mm})$ can be selected in order to minimize the measuring time (Fig. 6b).

In conclusion, workpiece measurements by means of the touch probe should be carried out according to the following procedure:

1) calibrate the touch probe on a reference surface using the suggested measuring parameters (feed rate $=150 \mathrm{~mm} / \mathrm{min}$, stand-off distance $=1 \mathrm{~mm}$ );

2) perform a first measure of the target surface using any standard stand-off distance and set the rough surface coordinate;

3) move the touch probe to the suggested stand-off distance and perform a new measurement, which will be the accurate one.

\section{Results}

\subsection{Set-up procedure}

Summarizing the aforementioned results, the following actions have to be performed prior to machining in order to minimize errors in absolute coordinates:

1) check and correct any malfunctioning of the machine (Section 3);

2) accurately design fixturing system, cutting parameters and milling strategies (Section 3 )

3) exhaust any thermal transient effects (Section 4.1);

4) set the Marposs VTS ${ }^{\odot}$ origin by a calibrated pin before measuring the selected mill (Section 4.2);

5) follow the procedure described in Section 4.3 for new origin setting and workpiece measurements.

\subsection{Workpiece accuracy}

The benchmark workpiece was machined after applying the proposed procedure. The specimen was measured onboard by means of the m\&h 32.00-MINI touch probe (Fig. 7). Both the workpiece and the slot surfaces were measured in random positions and an error of $\pm 1 \mu \mathrm{m}$ was detected. The comparison between this result and the error measured in the past study [6-7] demonstrates the effectiveness of the designed procedure to machine accurate plane surfaces with a strict flatness deviation.

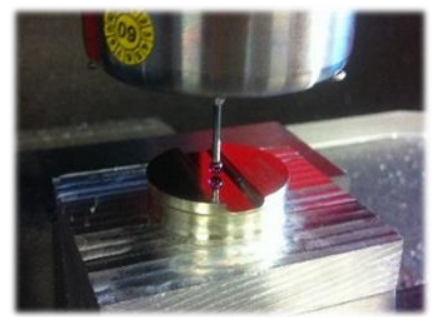

Fig. 7. Onboard measurements of machined benchmark workpiece.

\section{Conclusions and further developments}

The present paper proposes an easy and industry-oriented procedure to maximize the achievable workpiece accuracy working in absolute coordinates. Onboard measurements on the benchmark workpiece demonstrated the procedure effectiveness.

Further developments of the present study will include the complete characterization of the touch probe along $X$ and $Y$ axis. Moreover, further experiments will be performed concerning the $Z$ axis to exclude possible time transients. Eventually, further studies will aim at measuring the forces generated by the probing operations on a stiff workpiece. Pin and thin walls probing operations could also be investigated to characterize the sensor behaviour when measuring deformable features.

\section{References}

[1] D. Dornfeld et al., "Recent advances in mechanical micromachining", CIRP Ann-Manuf. Technol., 2006; 55: 745-768.

[2] G. Byrne et al., Advancing cutting technology, CIRP Ann-Manuf. Technol., 2003; 52: 483-507.

[3] J. Chae et al., "Investigation of micro-cutting operations", Int. J. Mach. Tools Manuf., 2006; 46: 313-332.

[4] K. Popov et al., "New tool-workpiece setting up technology for micro-milling", Int. J. Adv. Manuf. Technol., 2010; 47: 21-27.

[5] J.R. Mayor et al., "Intelligent tool-path segmentation for improved stability and reduced machining time in micromilling", J. Manuf. Sci. Eng.-Trans. ASME, 2008; 130.

[6] M. Annoni et al., "Workpiece surface flatness improvement by tool length compensation in micromilling", ICOMM 2014 Conference. \#107.

[7] A. Davoudinejad et al., "Improvement of surface flatness in high precision milling", Euspen 2014 Conference. 190-193. 